\title{
Clinical utility gene card for: MAN1B1 defective congenital disorder of glycosylation
}

\author{
Jaak Jaeken $^{\star, 1}$, Dirk J Lefeber ${ }^{2}$ and Gert Matthijs ${ }^{3}$ \\ European Journal of Human Genetics (2016) 24, doi:10.1038/ejhg.2015.248; published online 18 November 2015
}

\section{DISEASE CHARACTERISTICS}

1.1 Name of the disease (synonyms)

Deficiency of Golgi mannosyl-oligosaccharide $\alpha 1,2$-mannosidase, MAN1B1 deficiency, MAN1B1-CDG.

\subsection{OMIM\# of the Disease \\ 614202.}

1.3 Name of the Analysed Gene or DNA/Chromosome Segments MAN1B1.

\subsection{OMIM\# of the Gene(s)}

604346 .

\subsection{Mutational Spectrum}

Nineteen variants have been reported, including 11 missense variants, 1 nonsense variant, 5 deletion variants, and 2 splicing variants, but the clinical significance is not fully clarified for most of these variants. ${ }^{1-3}$ (www.lovd.nl/MAN1B1). The standard reference sequence indicating reported variants (ENSG00000177239) and a reference for exon numbering (ENST00000474902) can be found at http://www. ensembl.org.

\subsection{Analytical Methods}

Sanger sequencing of the 13 coding exons and flanking intronic sequences of the MAN1B1 gene (NCBI reference sequence: NM_016219.4).

\subsection{Analytical Validation}

Sanger sequencing identifies variants in $>99 \%$ of patients. Deep intronic variants, large deletions, and duplications would not be detected using this approach. Novel variants with uncertain pathogenic nature are of course possible.

\subsection{Estimated Frequency of the Disease}

(Incidence at birth ('birth prevalence') or population prevalence) if known to be variable between ethnic groups, please report):

Thirty-one patients (from 20 families) have been reported. ${ }^{1-3}$ The frequency and the prevalence of the disease are not known.

\subsection{Diagnostic Setting}

\begin{tabular}{lcc}
\hline & Yes. & No. \\
A. (Differential) diagnostics & $\bigotimes$ & \\
B. Predictive testing & $\bigotimes$ & \\
C. Risk assessment in relatives & $\bigotimes$ & $\square$ \\
D. Prenatal & $\bigotimes$ & $\square$
\end{tabular}

Comment: MAN1B1-CDG is an autosomal recessive disorder. All reported patients showed intellectual/developmental disability, ranging from mild to severe. Most patients also presented abnormal speech development and hypotonia. ${ }^{1-3}$ In the majority of patients there was facial dysmorphism (mainly down-slanting palpebral fissures, prominent eyebrows with lateral thinning, prominent bulbous nose tip, tent-shaped mouth with thin upper lip, and large ears) and truncal obesity. Behavioural problems have been reported in about half of the patients, particularly verbal and physical aggression, inappropriate sexual behaviour and overeating. A subset of patients showed mild dolichocephaly, long and thin fingers, and increased skin laxity and joint hyperlaxity. No clear phenotype-genotype correlations could be found. Biochemical abnormalities such as increased serum transaminases and abnormal coagulation tests were present in only a few patients. Serum transferrin isoelectrofocusing shows a type 2 pattern. Mass spectrometry of transferrin shows a specific accumulation of hybrid type $\mathrm{N}$-glycans. The diagnosis has to be confirmed by mutation analysis of MAN1B1. An upcoming strategy, after finding and confirming an abnormal serum transferrin isoelectrofocusing pattern, is to subject the DNA to a CDG panel of genes known to be involved in CDG. The identification of the pathogenic variant will permit heterozygote detection in the family, and prenatal diagnosis.

\section{TEST CHARACTERISTICS}

\begin{tabular}{|c|c|c|c|c|}
\hline & \multicolumn{2}{|c|}{ Genotype or disease } & \multirow{2}{*}{$\begin{array}{l}\text { A: true positives } \\
\text { B: false positives }\end{array}$} & \multirow{2}{*}{$\begin{array}{l}\text { C: false negative } \\
\text { D: true negative }\end{array}$} \\
\hline & Present & Absent & & \\
\hline \multicolumn{5}{|l|}{ Test } \\
\hline Pos. & A & B & $\begin{array}{l}\text { Sensitivity: } \\
\text { Specificity: }\end{array}$ & $\begin{array}{l}A /(A+C) \\
D /(D+B)\end{array}$ \\
\hline Neg. & C & D & $\begin{array}{l}\text { Pos. predict. value: } \\
\text { Neg. predict. value: }\end{array}$ & $\begin{array}{l}A /(A+B) \\
D /(C+D)\end{array}$ \\
\hline
\end{tabular}

\footnotetext{
${ }^{1}$ Centre for Metabolic Disease, University Hospital Gasthuisberg, Leuven, Belgium; ${ }^{2}$ Department of Neurology, Translational Metabolic Laboratory, Radboudumc, Nijmegen, The Netherlands; ${ }^{3}$ Centre for Human Genetics, KULeuven, Leuven, Belgium

*Correspondence: Professor J Jaeken, Centre for Metabolic Disease, University Hospital Gasthuisberg, Herestraat 49 , BE 3000 Leuven, Belgium. Tel: +32 16343827 ; Fax: +32 16 343842; E-mail: jaak.jaeken@med.kuleuven.be

Received 13 July 2015; revised 6 October 2015; accepted 21 October 2015; published online 18 November 2015
} 
2.1 Analytical Sensitivity

(proportion of positive tests if the genotype is present)

Close to $100 \%$ when using the serum transferrin isoelectrofocusing test.

\subsection{Analytical Specificity}

(proportion of negative tests if the genotype is not present)

Close to $100 \%$ when using the serum transferrin isoelectrofocusing test. This test can be positive in secondary glycosylation disturbances such as galactosemia and hereditary fructose intolerance, and due to bacterial sialidase. ${ }^{4-6}$

\subsection{Clinical Sensitivity}

(proportion of positive tests if the disease is present)

The clinical sensitivity can be dependent on variable factors such as age or family history. In such cases a general statement should be given, even if a quantification can only be made case by case.

Close to $100 \%$.

\subsection{Clinical Specificity}

(proportion of negative tests if the disease is not present)

The clinical specificity can be dependent on variable factors such as age or family history. In such cases a general statement should be given, even if a quantification can only be made case by case.

Close to $100 \%$.

2.5 Positive clinical predictive value

(life-time risk to develop the disease if the test is positive)

$100 \%$, based on positive serum transferrin isoelectrofocusing screening and MAN1B1 mutation analysis.

2.6 Negative clinical predictive value

(Probability not to develop the disease if the test is negative)

Assume an increased risk based on family history for a nonaffected person. Allelic and locus heterogeneity may need to be considered.

Index case in that family had been tested:

$100 \%$.

Index case in that family had not been tested:

$100 \%$.

\section{CLINICAL UTILITY}

3.1 (Differential) diagnostics: The tested person is clinically affected

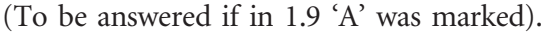

\subsubsection{Can a diagnosis be made other than through a genetic test?}

\begin{tabular}{lll}
\hline No. & $\square$ (continue with 3.1.4) \\
Yes, & $\bigotimes$ & \\
& Clinically & \\
& Imaging. & $\square$ \\
& Endoscopy. & $\square$ \\
& Biochemistry. & $\square$ \\
& Electrophysiology. & $\square$ \\
& Other (please describe)
\end{tabular}

3.1.2 Describe the burden of alternative diagnostic methods to the patient

The blood sampling for the serum transferrin isoelectrofocusing screening test and that for the mutation analysis is a minor burden to the patient.
3.1.3 How is the cost effectiveness of alternative diagnostic methods to be judged?

It differs among countries. In Belgium and The Netherlands the cost of these tests is largely carried by the national assurance organism.

3.1.4 Will disease management be influenced by the result of a genetic test?

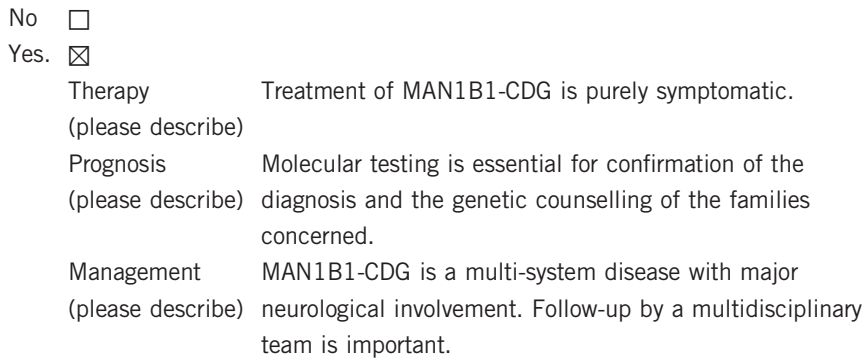

3.2 Predictive Setting: The tested person is clinically unaffected but carries an increased risk based on family history

(To be answered if in 1.9 'B' was marked).

3.2.1 Will the result of a genetic test influence lifestyle and prevention?

If the test result is positive (please describe).

Not applicable

If the test result is negative (please describe).

Not applicable.

3.2.2 Which options in view of lifestyle and prevention does a person at-risk have if no genetic test has been done (please describe)?

Not applicable.

3.3 Genetic risk assessment in family members of a diseased person

(To be answered if in 1.9 'C' was marked).

3.3.1 Does the result of a genetic test resolve the genetic situation in that family?

Usually yes, by testing the potential heterozygous persons (carriers) in the family.

3.3.2 Can a genetic test in the index patient save genetic or other tests in family members?

No.

3.3.3 Does a positive genetic test result in the index patient enable a predictive test in a family member?

Not applicable.

3.4 Prenatal diagnosis

(To be answered if in 1.9 ' $\mathrm{D}$ ' was marked).

3.4.1 Does a positive genetic test result in the index patient enable a prenatal diagnosis?

Yes. Prenatal diagnosis should be performed by molecular analysis.

\section{IF APPLICABLE, FURTHER CONSEQUENCES OF TESTING}

Please assume that the result of a genetic test has no immediate medical consequences. Is there any evidence that a genetic test is nevertheless useful for the patient or his/her relatives? (Please describe) 
Knowledge of the diagnosis will stop unnecessary further investigations, and will help the parents in the process of accepting the disease although no curative treatment is available.

\section{CONFLICT OF INTEREST}

The authors declare no conflict of interest.

\section{ACKNOWLEDGEMENTS}

This work was supported by EuroGentest2 (unit 2: 'Genetic testing as part of health care'), a Coordination Action under FP7 (Grant Agreement No 261469) and the European Society of Human Genetics.
1 Rafiq MA, Kuss AW, Puettmann L et al: Mutations in the alpha 1,2-mannosidase gene, MAN1B1, cause autosomal-recessive intellectual disability. Am J Hum Genet 2011; 89: 176-182.

2 Rymen D, Peanne R, Millón MB et al: MAN1B1 deficiency: an unexpected CDG-II. PLoS Genet 2013; 9 (12): e1003989.

3 Van Scherpenzeel M, Timal S, Rymen D et al: Diagnostic serum glycosylation profile in patients with intellectual disability as a result of MAN1B1 deficiency. Brain 2014; 137 : 1030-1038.

4 Jaeken J, Pirard M, Adamowicz M, Pronicka E, Van Schaftingen E: Inhibition of phosphomannose isomerase by fructose 1-phosphate: an explanation for defective $\mathrm{N}$-glycosylation in hereditary fructose intolerance. Pediatr Res 1996; 40: 764-766.

5 Sturiale L, Barone R, Fiumara A et al: Hypoglycosylation with increased fucosylation and branching of serum transferrin N-glycans in untreated galactosemia. Glycobiology 2005; 15: $1268-1276$.

6 Lefeber DJ, Morava E, Jaeken J: How to find and diagnose a CDG due to defective N-glycosylation. J Inherit Metab Dis 2011; 34: 849-852. 